\title{
El gasto público en medio ambiente: un análisis comparativo del caso de España y Andalucía.
}

\author{
Macarena LOZANO OYOLA*
}

\section{RESUMEN}

En este artículo analizaremos la evolución registrada por el Gasto Público en medio ambiente en España respecto al Gasto Público total entre 1987 y 2004. Para ello estudiaremos a nivel nacional las partidas que componen el Gasto Público en medio ambiente, según diferentes clasificaciones.

Una vez que conocemos la evolución en el ámbito nacional, analizaremos las cifras de Gasto Público en medio ambiente de la Comunidad Autónoma de Andalucía. Se estudiarán la participación del Gasto Público en medio ambiente en el total de Gasto Público de la Junta de Andalucía, los diferentes programas puestos en marcha y la evolución de los capítulos presupuestarios. Por último, se realizará un análisis comparativo de las cifras obtenidas en el ámbito andaluz y nacional.

\section{ABSTRACT}

In this article we will analyze the evolution of the environmental Public Expenses in Spain regarding total Public Expenses between 1987 and 2004. With that purpose, we will study at national level the different concepts included in environmental Public Expenses, according to different classifications.

Once we know the evolution in the whole country, we will analyze the figures of the environmental Public Expenses of the Andalusian Regional Government.

\footnotetext{
*Profesora de la Universidad Pablo de Olavide (Sevilla)
} 
We will study the participation of the environmental Public Expenses in the total Public Expenses of the Andalusian Regional Government, the different programs implemented and the evolution of the budgetary chapters. Finally, a comparative analysis of the figures obtained in the Andalusian and whole country will be carried out.

\section{INTRODUCCIÓN}

En este artículo analizaremos la evolución registrada por el Gasto Público en medio ambiente en España respecto al Gasto Público total entre 1987 y 2004. Para ello estudiaremos a nivel nacional las partidas que componen el Gasto Público en medio ambiente, según diferentes clasificaciones (actividades características medioambientales, capítulos presupuestarios y sectores institucionales). Dividiremos el periodo objeto de estudio en dos: para el periodo 1987-95 tomaremos la información proporcionada por el Ministerio de Medio Ambiente y para los años 1996-2004 se recurrirá a los Presupuestos Generales del Estado, ya que los datos posteriores a 1995 no han sido presentados por el Ministerio de Medio Ambiente.

Una vez que se conoce la evolución a nivel nacional, nos centraremos en el estudio de las cifras de Gasto Público en medio ambiente de la Comunidad Autónoma de Andalucía. Serán analizados tanto la participación del Gasto Público en medio ambiente en el total de Gasto Público de la Junta de Andalucía como los diferentes programas puestos en marcha y la evolución de los capítulos presupuestarios. Por último, se realizará un análisis comparativo de las cifras obtenidas en el ámbito andaluz y nacional.

\section{GASTO PÚBLICO EN MEDIO AMBIENTE EN ESPAÑA}

Para analizar la importancia del Gasto Público en medio ambiente en España vamos a partir de la incorporación a la Comunidad Económica Europea, momento en el que nuestro país presentaba un elevado déficit en materia ambiental. Desde entonces, el Gasto Público en España en medio ambiente ha mantenido un crecimiento anual considerable tanto en el conjunto del Gasto Público como en el PIB. En la Cuadro 9 aparecen los ratios, índices y tasas de variación del Gasto Público en medio ambiente. 


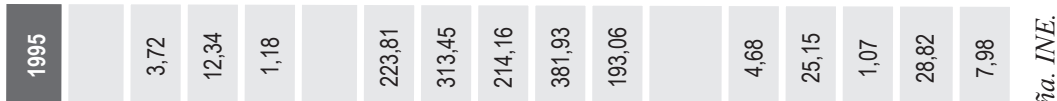

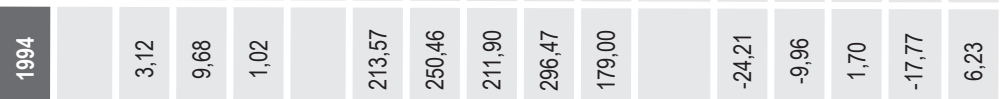

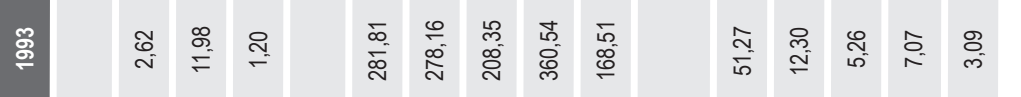

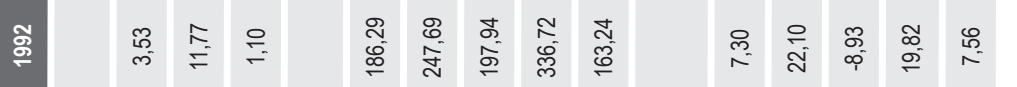

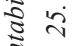

ฐัฐ

항 $\sqrt{2}$

:

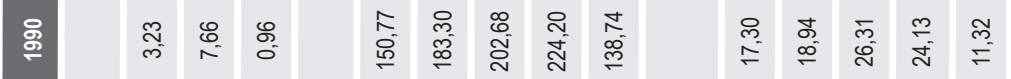

m m

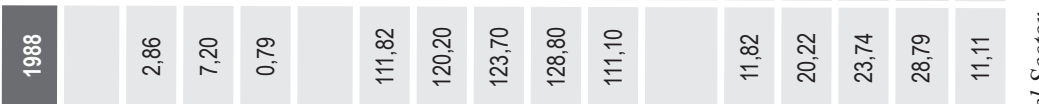

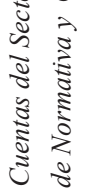

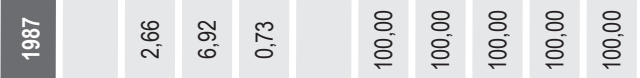

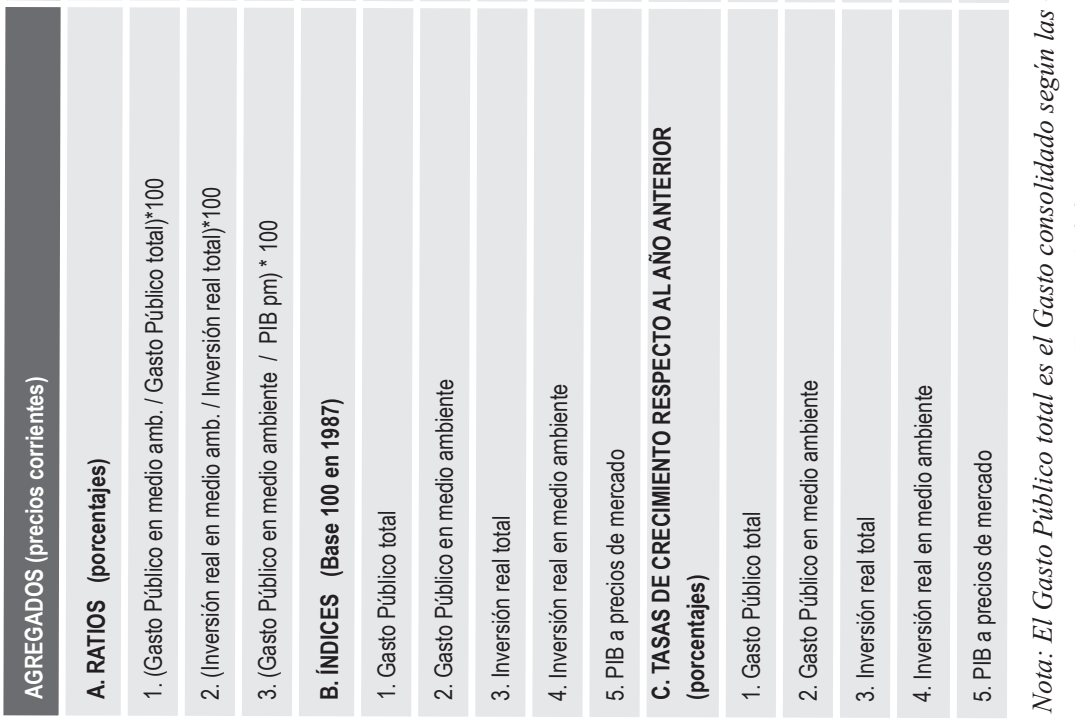


Observando los datos en el periodo analizado (años 1987 a 1995), podemos llegar a las siguientes conclusiones: un aumento de la importancia del Gasto Público en medio ambiente respecto al Gasto Público total a lo largo del periodo y un incremento de la Inversión real en medio ambiente superior al experimentado por la Inversión real total.

Por lo que respecta al ratio que relaciona el Gasto Público en medio ambiente con el PIB, hay que destacar cómo en 1995 se recupera tras la bajada que sufre en 1994. Un comportamiento similar observamos en el ratio referido a la Inversión real y al Gasto Público (en este caso la bajada se produce en 1993). En los Gráficos 4 y 5 se refleja la evolución del Gasto Público, la Inversión real y el PIB a precios de mercado.

Gráfico 4. Evolución del gasto público en medio ambiente, el gasto público total y el pib.

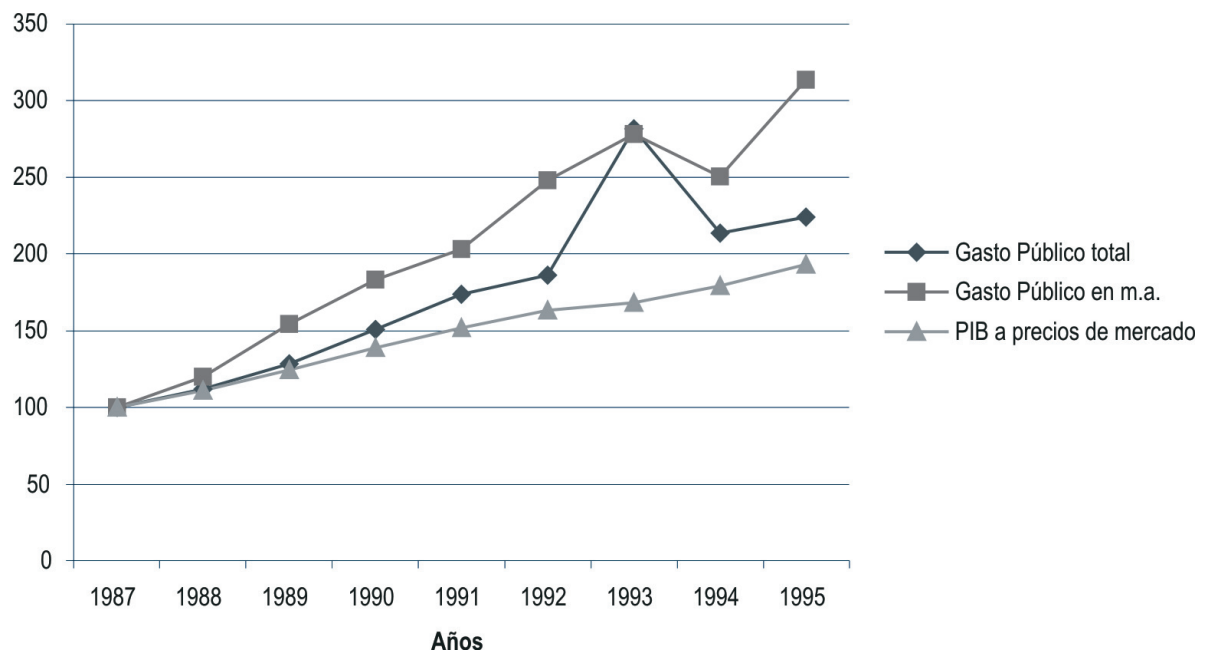

Nota: Año $1987=100$

Fuente: Subdirección General de Normativa y Cooperación Institucional (1998) y elaboración propia. 
Gráfico 5. Evolución de la inversión real en medio ambiente, la inversión real total y el pib.

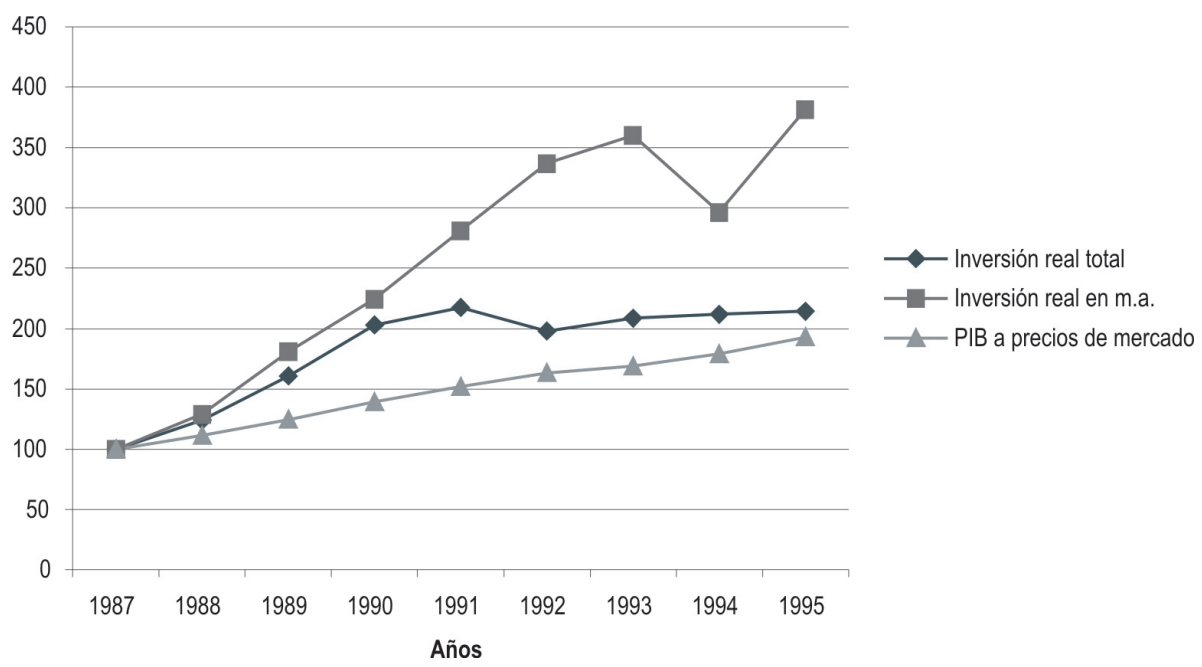

Nota: Año $1987=100$

Fuente: Subdirección General de Normativa y Cooperación Institucional (1998) y elaboración propia.

Por lo que se refiere a la evolución del gasto de las Administraciones Públicas ${ }^{1}$, ésta puede ser analizada en función de varios criterios. En el Gráfico 6 aparece la evolución del Gasto consolidado de las Administraciones Públicas en Actividades características: protección del medio ambiente, y uso y gestión de los recursos naturales. En cuanto al reparto entre estas actividades, queda reflejado cómo la protección del medio ambiente representa entre un $75 \%$ y un $80 \%$ entre 1987 y 1995 , mientras que el uso y la gestión de los recursos naturales oscila entre un $25 \%$ y un $20 \%$.

El Gasto consolidado del Sector Público se ha obtenido para cada uno de los sectores y subsectores institucionales descontando las transferencias internas, tanto corrientes como de capital. 
Gráfico 6. Gasto consolidado de las administraciones públicas en actividades características medioambientales, por actividades características: estructura porcentual.

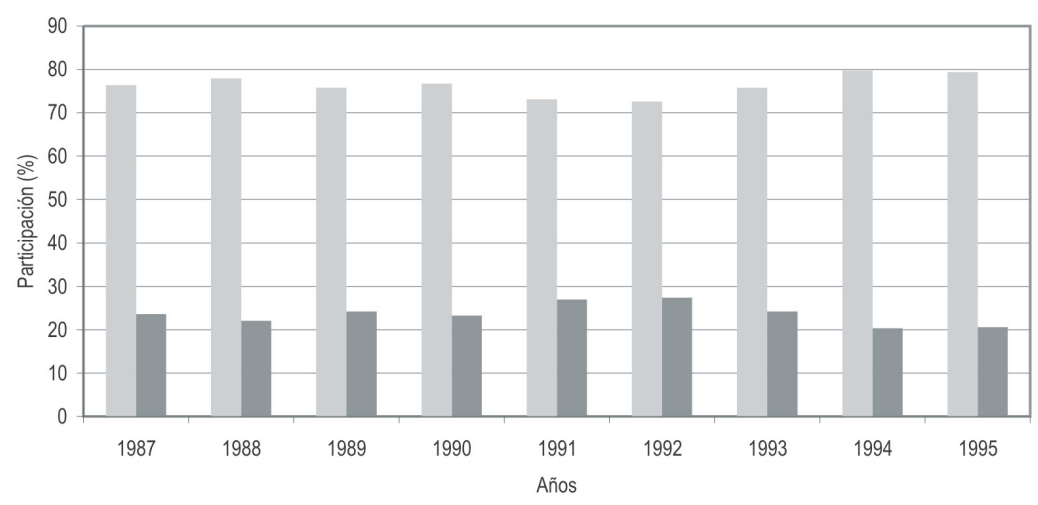

- PROTECCIÓN DEL MEDIO AMBIENTE —USO Y GESTIÓN DE LOS RECURSOS NATURALES

Fuente: Subdirección General de Normativa y Cooperación Institucional (1998), págs. 161-162, y elaboración propia.

Si analizamos la distribución del Gasto consolidado de las Administraciones Públicas por capítulos presupuestarios (Cuadro 10), debemos distinguir entre los gastos corrientes ${ }^{2}$ y los gastos de capital ${ }^{3}$. Analizando su comportamiento en el tiempo, observamos una reducción constante de los gastos corrientes. La disminución corresponde a las partidas de gastos de personal (que pasa del 23\% a un 16,86\%) y de compras de bienes y servicios (desciende desde un $42 \%$ en 1987 al $25,36 \%$ de 1995), aumentando levemente los gastos financieros y en mayor medida las transferencias corrientes (se multiplican por diez).

Durante este periodo, los gastos de capital experimentan un incremento, siendo la partida más importante la correspondiente a las inversiones reales $(39,9 \%$ en 1995).

\footnotetext{
${ }^{2}$ Incluyen los gastos de personal, los gastos de bienes y servicios, los gastos financieros y las transferencias corrientes.

${ }^{3}$ Comprenden las inversiones reales y las transferencias de capital.
} 


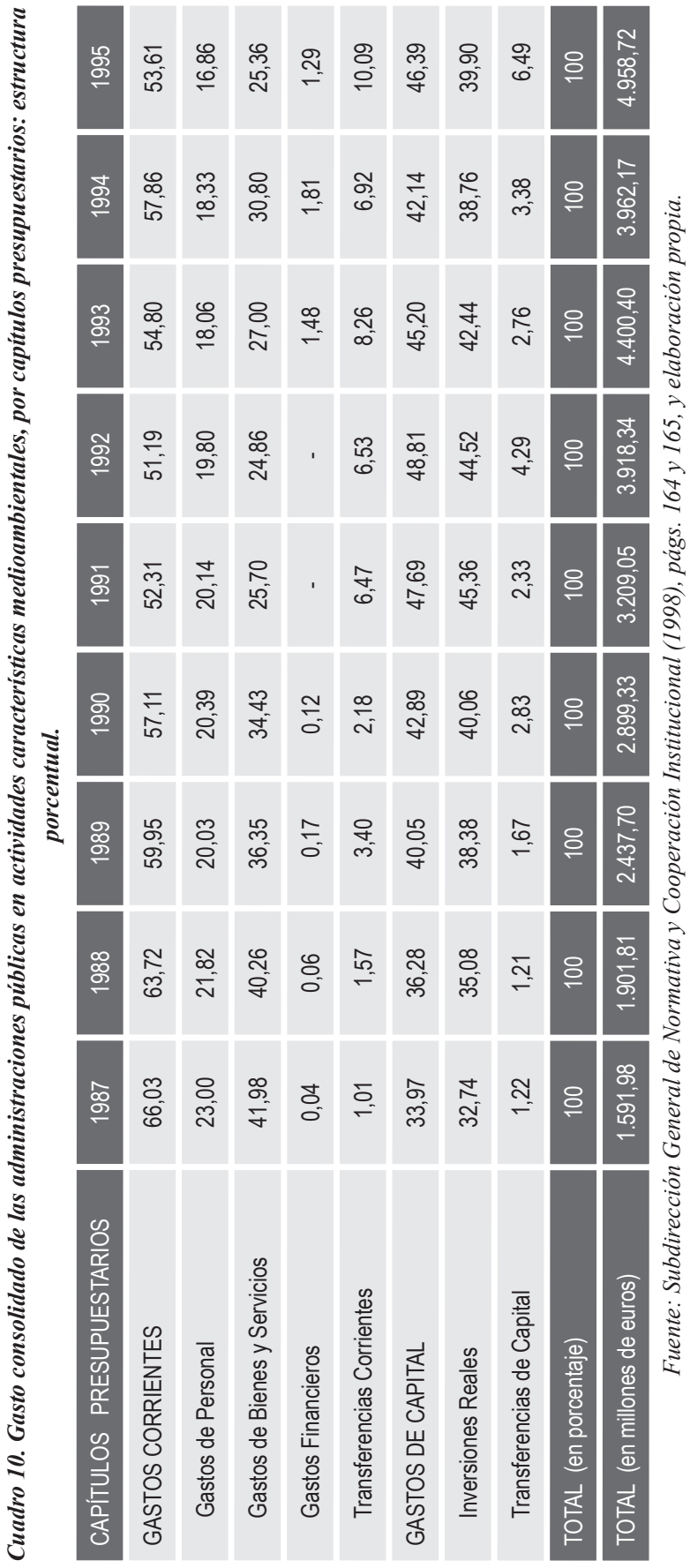


Por lo que se refiere a las transferencias a otros agentes económicos ${ }^{4}$, es necesario destacar que se produce un aumento muy significativo tanto en el caso de las transferencias corrientes como en las de capital.

Una vez que hemos analizado la información relativa al Gasto Público en medio ambiente proporcionada por el Ministerio de Medio Ambiente (1987-95), vamos a actualizar estos datos. Para ello, y dado que los datos de los años 19962003 no han sido presentados por el Ministerio de Medio Ambiente, debemos recurrir a los Presupuestos Generales del Estado. Para comparar esta información con la proporcionada por el Ministerio de Medio Ambiente (1987-95), sería conveniente contar con la función de Gasto en Medio Ambiente realizada por las diferentes Secciones. Dado que ésta no aparece recogida como tal en los Presupuestos Generales del Estado, habremos de trabajar con el Gasto Público consolidado de la Sección 23 Ministerio de Medio Ambiente, aun sabiendo que otras Secciones como el Ministerio de Agricultura o el de Fomento destinan partidas a las cuestiones ambientales.

Para los años 1997 a 2004 contamos con una información homogénea. En el año 1996, y dado que no se aprobó el Presupuesto, fue prorrogado el del año anterior. En el año 1995 no existía Ministerio de Medio Ambiente y la Sección de Gasto más similar es el Ministerio de Obras Públicas, Transportes y Medio Ambiente. Dicho Ministerio contaba entre sus subsecciones con la Secretaría de Estado de Medio Ambiente y Vivienda (lo que distorsiona el análisis), la Dirección General de la Calidad del Agua, la Dirección General de Política Ambiental, la Dirección General de Costas, la Dirección General del Instituto de Meteorología y la Dirección General de Obras Hidráulicas, que han pasado a formar parte, aunque con modificaciones en algunas denominaciones, del Ministerio de Medio Ambiente. En primer lugar, analizaremos el Gasto Público de la Sección 23 Ministerio de Medio Ambiente y del Subsector Estado, por capítulos presupuestarios.

\footnotetext{
${ }^{4}$ Seguridad Social, Empresas Públicas, Empresas Privadas, Familias e Instituciones Privadas y Exterior.
} 


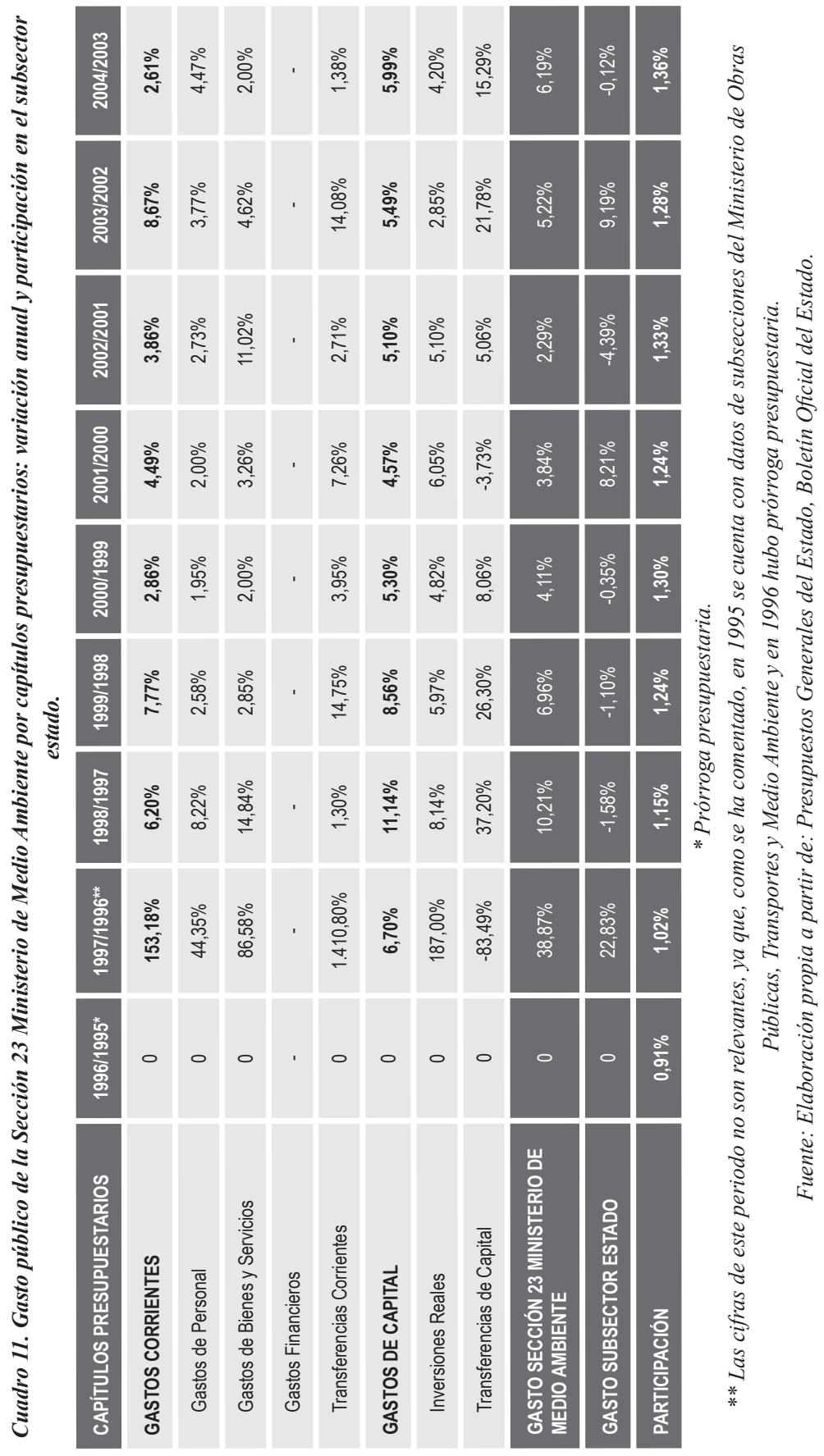


Cuando se analiza la variación anual del Gasto de la Sección 23, lo primero a tener en cuenta es que no deben tomarse en consideración las cifras relativas a la variación experimentada entre los años 1996 y 1997. Las distorsiones en el análisis resultan muy importantes, dado que se produjo un cambio de Gobierno y por el hecho de que el año anterior hubiera una prórroga presupuestaria.

Si comparamos la evolución seguida por el Gasto en medio ambiente por capítulos presupuestarios (Cuadro 115), debemos destacar la mayor tendencia alcista de los gastos corrientes frente a los gastos de capital. Además, podemos observar cómo en el periodo 1997-2004 el crecimiento de la Sección Medio Ambiente supera el registrado por el Subsector Estado. Esto conlleva un incremento de la participación del Medio Ambiente en los Presupuestos Generales del Estado (Gráfico 7).

Gráfico 7. Participación del gasto de la Sección 23 Ministerio de Medio Ambiente en el subsector estado.

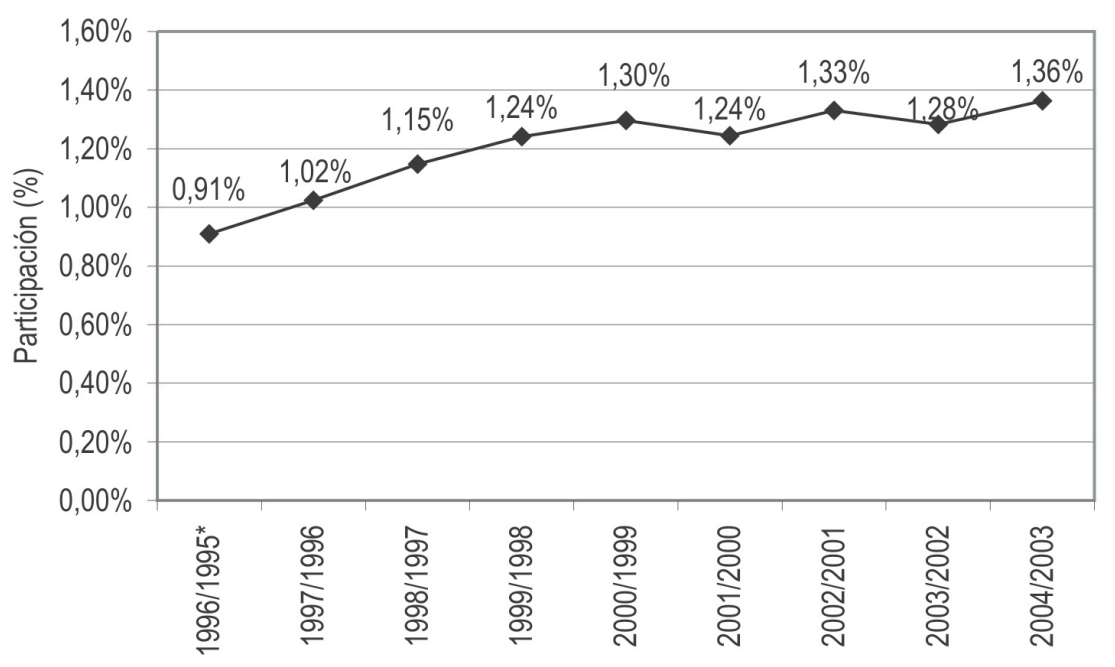

Fuente: Elaboración propia a partir de: Presupuestos Generales del Estado, Boletín Oficial del Estado.

Por lo que se refiere al Gasto consolidado (Gráfico 8 y Cuadro 12), tanto el relativo al medio ambiente como el total registran un crecimiento que es mucho mayor en el caso del medio ambiente. La participación a lo largo de los años 1997-2004 experimenta, por tanto, un aumento.

\footnotetext{
${ }^{5}$ Se ha eliminado la fila correspondiente a los gastos financieros, ya que la variación anual carecería de sentido.
} 
Gráfico 8. Gasto consolidado de la sección 23 ministerio de medio ambiente y total: participación.

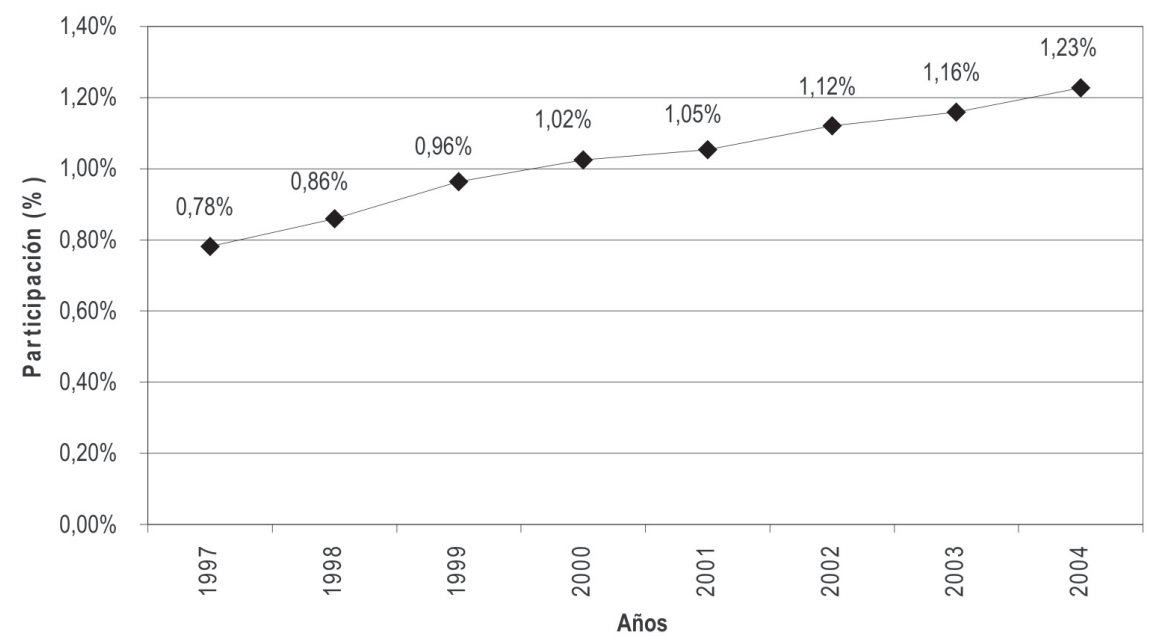

Fuente: Elaboración propia a partir de: Presupuestos Generales del Estado, Boletín Oficial del Estado.

Cuadro 12. Gasto consolidado de la Sección 23 Ministerio de Medio Ambiente y total: valor absoluto, variación anual y participación.

\begin{tabular}{|c|c|c|c|c|}
\hline GASTO CONSOLIDADO & 1997 & 1998 & 1999 & 2000 \\
\hline \multicolumn{5}{|l|}{ MEDIO AMBIENTE } \\
\hline GASTO CONSOLIDADO & 1.657 .984 & 1.825 .980 & 2.065 .680 & 2.236 .188 \\
\hline TASA DE CRECIMIENTO & - & $10,13 \%$ & $13,13 \%$ & $8,25 \%$ \\
\hline \multicolumn{5}{|l|}{ TOTAL } \\
\hline GASTO CONSOLIDADO & 212.213.011 & 212.355 .165 & 214.455 .386 & 218.188 .235 \\
\hline TASA DE CRECIMIENTO & - & $0,07 \%$ & $0,99 \%$ & $1,74 \%$ \\
\hline PARTICIPACIÓN & $0,78 \%$ & $0,86 \%$ & $0,96 \%$ & $1,02 \%$ \\
\hline GASTO CONSOLIDADO & 2001 & 2002 & 2003 & 2004 \\
\hline \multicolumn{5}{|l|}{ MEDIO AMBIENTE } \\
\hline GASTO CONSOLIDADO & 2.494 .238 & 2.678 .623 & 3.001 .682 & 3.253 .479 \\
\hline TASA DE CRECIMIENTO & $11,54 \%$ & $7,39 \%$ & $12,06 \%$ & $8,39 \%$ \\
\hline \multicolumn{5}{|l|}{ TOTAL } \\
\hline GASTO CONSOLIDADO & 236.781 .830 & 238.919 .822 & 258.843 .068 & 264.961 .709 \\
\hline TASA DE CRECIMIENTO & $8,52 \%$ & $0,90 \%$ & $8,34 \%$ & $2,36 \%$ \\
\hline PARTICIPACIÓN & $1,05 \%$ & $1,12 \%$ & $1,16 \%$ & $1,23 \%$ \\
\hline
\end{tabular}

Datos en miles de euros.

Fuente: Elaboración propia a partir de: Presupuestos Generales del Estado, Boletín Oficial del Estado. 


\section{GASTO PÚBLICO EN MEDIO AMBIENTE DE ANDALUCÍA}

Para analizar el Gasto Público en medio ambiente de Andalucía vamos a estudiar el Presupuesto de la Comunidad Autónoma de Andalucía, así como los diferentes Programas que componen la Política de medio ambiente. Al igual que hemos hecho en el caso del Gasto Publico a nivel nacional, no se tendrán en cuenta los gastos que en materia de medio ambiente puedan realizar otros organismos, tales como la Consejería de Agricultura.

Partimos de la base de que Andalucía es una Comunidad Autónoma que cuenta con una de las mayores diversidades biológicas de Europa: en su territorio se encuentran más de 400 especies de animales y 4.000 especies de plantas. Esto conlleva que una parte importante de su territorio se encuentre protegida, concretamente el $17 \%$ de la superficie total ${ }^{6}$. Junto a este hecho, debemos hacer referencia a los impactos ambientales causados por actividades económicas como la agricultura intensiva que aparece en la mayor parte del litoral, la concentración de industrias muy contaminantes en determinadas zonas como la Ría de Huelva y la Bahía de Algeciras, así como la actividad turística caracterizada por su intensidad y falta de planificación.

Con la intención de aprovechar las potencialidades del medio y corregir los déficits ambientales, se creó en 1984 la Agencia de Medio Ambiente, el primer organismo de estas características creado en España, y diez años más tarde, la Consejería de Medio Ambiente. Aunque la Comunidad Autónoma andaluza tiene transferidas las competencias en materia de medio ambiente ${ }^{7}$ desde 1984 , la mayor parte de los datos a los que vamos a hacer referencia parten del año 1987 o de principios de los noventa, ya que estas fechas son las analizadas a nivel nacional.

Por lo que se refiere a los datos de Gasto Público en Medio Ambiente que aparecen en las tablas, debemos decir que entre 1987 y 1993 corresponden al Presupuesto de la Agencia de Medio Ambiente, y a partir de 1994, a la Consejería de Medio Ambiente de la Junta de Andalucía.

\footnotetext{
${ }^{6}$ Este porcentaje se sitúa en el $7 \%$ en España y en el 7,7\% en la OCDE.

${ }^{7}$ Con la excepción de las aguas continentales que están a cargo de las Confederaciones Hidrográficas.
} 
Antes de iniciar el comentario de las cifras, en las diferentes tablas, debemos tener en cuenta que en 1995 el Presupuesto de la Junta de Andalucía fue prorrogado y que en 1996 existió una prórroga presupuestaria a nivel nacional, por lo que en la realidad se les asignó menos dinero del realmente presupuestado ${ }^{8}$.

En la Cuadro 13aparece la Política de Medio Ambiente y el total presupuestario de Andalucía. Podemos decir que la dotación presupuestaria que la Junta de Andalucía dedica a las actuaciones medioambientales representa un porcentaje pequeño con relación al volumen total del Presupuesto, aunque en los últimos años esta partida ha experimentado un importante crecimiento: si tomamos los valores absolutos podemos comprobar que mientras que el Presupuesto de la Junta de Andalucía entre 1987 y 2003 se multiplica por menos de cinco, el Gasto Público en medio ambiente se multiplica por dieciséis.

Cuadro 13. Evolución de la política de medio ambiente y del total presupuestario de la Comunidad Autónoma de Andalucía.

\begin{tabular}{|l|c|c|c|c|c|c|c|c|c|c|}
\hline POLítICAS & 1987 & 1988 & 1989 & 1990 & 1991 & 1992 & 1993 & 1994 & 1995 & 1996 \\
\hline $\begin{array}{l}\text { Gasto Público en } \\
\text { Medio Ambiente }\end{array}$ & 23 & 29 & 46 & 60 & 81 & 107 & 108 & 112 & 225 & 233 \\
\hline Gasto Público total & 4.069 & 4.802 & 6.184 & 7.302 & 8.108 & 9.117 & 9.875 & 10.818 & 11.269 & 12.219 \\
\hline PARTICIPACIÓN (\%) & $\mathbf{0 , 5 8 \%}$ & $\mathbf{0 , 6 1 \%}$ & $\mathbf{0 , 7 5 \%}$ & $\mathbf{0 , 8 2 \%}$ & $\mathbf{0 , 9 9 \%}$ & $\mathbf{1 , 1 7 \%}$ & $\mathbf{1 , 1 0 \%}$ & $\mathbf{1 , 0 3 \%}$ & $\mathbf{2 , 0 0 \%}$ & $\mathbf{1 , 9 0 \%}$ \\
\hline
\end{tabular}

\begin{tabular}{|l|c|c|c|c|c|c|c|}
\hline POLÍTICAS & 1997 & 1998 & 1999 & 2000 & 2001 & 2002 & 2003 \\
\hline Gasto Público en Medio Ambiente & 216 & 231 & 265 & 290 & 304 & 340 & 370 \\
\hline Gasto Público total & 13.715 & 14.809 & 15.512 & 16.314 & 17.115 & 18.349 & 19.716 \\
\hline PARTICIPACIÓN (\%) & $1,57 \%$ & $1,56 \%$ & $1,71 \%$ & $1,78 \%$ & $1,77 \%$ & $1,85 \%$ & $1,88 \%$ \\
\hline
\end{tabular}

Datos en millones de euros.

Fuente: Elaboración propia a partir de Dirección General de Planificación y Participación, Informe Medio Ambiente en Andalucía, Junta de Andalucía, Sevilla, varios años y del Boletín Oficial de la Junta de Andalucía.

\footnotetext{
${ }^{8}$ Concretamente en 1995 las cifras presupuestadas (en millones de euros) fueron 231,39 para medio ambiente y 11.449,28 de Gasto Público total, frente a una liquidación de 225,38 y 11.268,98, respectivamente. En 1996 las cifras presupuestadas (en millones de euros) ascendían a 253,03 y 12.272,67, mientras que las cifras liquidadas se situaban en 232,59 y $12.218,58$, respectivamente.
} 
Al analizar la evolución del Gasto Público en medio ambiente se distinguen claramente cuatro periodos: entre 1987 y 1991 se multiplica el Gasto público en medio ambiente casi por cuatro; entre 1992 y 1994 se sitúa en torno a los 110 millones de euros; se duplica esta cifra en 1995 debido a la mayor importancia que se quiere prestar al entorno a través de la Consejería de Medio Ambiente alcanzando en el año 2000 casi los 300 millones de euros; en los últimos tres años el crecimiento es destacado, ya que se pasa de 304 a 370 millones de euros en dos años ${ }^{9}$. Esto se traduce en un aumento de la participación del Gasto Público en medio ambiente en el Gasto Público total, de manera que entre 1987 y 2003 la cifra ha aumentado en 1,3 puntos porcentuales.

Gráfico 9. Participación de la política de medio ambiente en el total presupuestario de la Comunidad Autónoma de Andalucía.

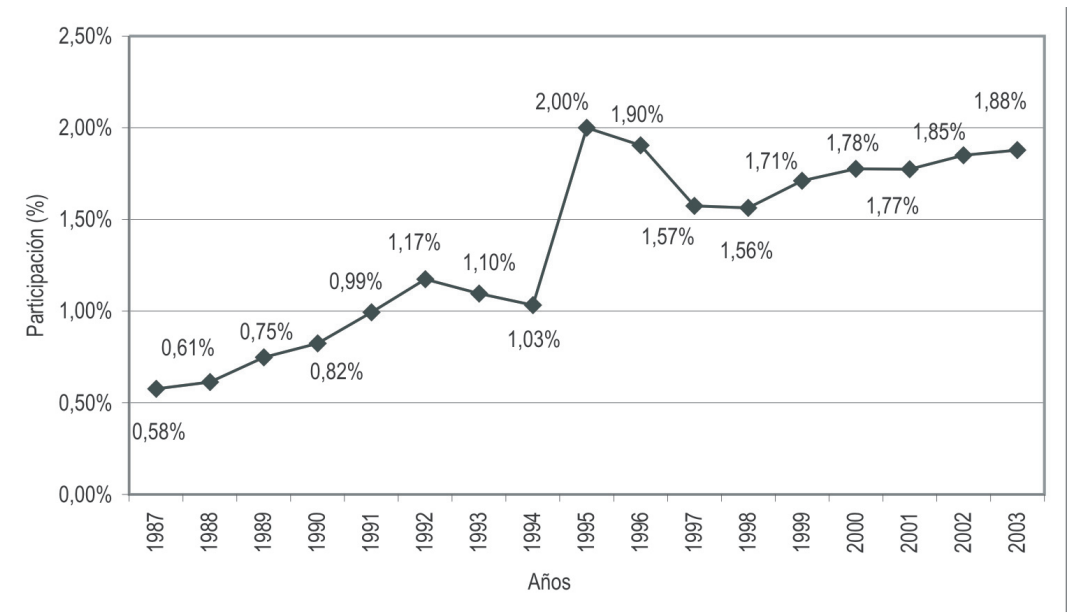

Fuente: Elaboración propia a partir de Dirección General de Planificación y Participación, Informe Medio Ambiente en Andalucía, Junta de Andalucía, Sevilla, varios años y del Boletín Oficial de la Junta de Andalucía.

\footnotetext{
${ }^{9}$ Como veremos más adelante, en 1999 la cifra es elevada porque recoge una partida de carácter excepcional, cercana a los 60,10 millones de euros, como consecuencia del vertido de residuos de la mina de Aznalcóllar en la Cuenca del Guadiamar.
} 
Cuadro 14. Tasas de crecimiento de la política de medio ambiente y del total presupuestario de la Comunidad Autónoma de Andalucía.

\begin{tabular}{|l|c|c|c|c|c|c|c|c|}
\hline CRECIMIENTO & 1987 & 1988 & 1989 & 1990 & 1991 & 1992 & 1993 & 1994 \\
\hline $\begin{array}{l}\text { Gasto Público en Medio } \\
\text { Ambiente }\end{array}$ & -- & $25,64 \%$ & $57,14 \%$ & $29,87 \%$ & $34,00 \%$ & $32,84 \%$ & $1,12 \%$ & $3,33 \%$ \\
\hline $\begin{array}{l}\text { Gasto Público de la Junta } \\
\text { de Andalucía }\end{array}$ & -- & $18,02 \%$ & $28,79 \%$ & $18,08 \%$ & $11,03 \%$ & $12,45 \%$ & $8,31 \%$ & $9,56 \%$ \\
\hline
\end{tabular}

\begin{tabular}{|l|c|c|c|c|c|c|c|c|c|}
\hline CRECIMIENTO & 1995 & 1996 & 1997 & 1998 & 1999 & $\mathbf{2 0 0 0}$ & $\mathbf{2 0 0 1}$ & $\mathbf{2 0 0 2}$ & 2003 \\
\hline $\begin{array}{l}\text { Gasto Público en } \\
\text { Medio Ambiente }\end{array}$ & $101,61 \%$ & $3,20 \%$ & $-7,24 \%$ & $7,24 \%$ & $14,63 \%$ & $9,25 \%$ & $4,77 \%$ & $11,82 \%$ & $9,10 \%$ \\
\hline $\begin{array}{l}\text { Gasto Público de la } \\
\text { Junta de Andalucía }\end{array}$ & $4,17 \%$ & $8,43 \%$ & $12,25 \%$ & $7,98 \%$ & $4,75 \%$ & $5,17 \%$ & $4,91 \%$ & $7,21 \%$ & $7,45 \%$ \\
\hline
\end{tabular}

Fuente: Elaboración propia a partir de Dirección General de Planificación y Participación, Informe Medio Ambiente en Andalucía, Junta de Andalucía, Sevilla, varios años y del Boletín Oficial de la Junta de Andalucía.

También resulta interesante comparar las tasas de crecimiento del Gasto Público en medio ambiente con las relativas al Gasto Público de la Junta de Andalucía (Cuadro 14). De ello se deduce que en el periodo 1987-92 se registra un crecimiento muy importante de las partidas dedicadas a las actuaciones medioambientales, muy superior al experimentado por el presupuesto autonómico. A partir de 1993 el crecimiento del Gasto Público en medio ambiente resulta en unos años superior al total del Gasto Público de la Junta de Andalucía, y en otros años inferior.

Si queremos estudiar cómo afecta el incremento global del presupuesto a los diferentes campos de intervención en materia de medio ambiente, debemos analizar la distribución del Presupuesto entre los años objeto de estudio. Para ello tomaremos los datos del Presupuesto de la Agencia de Medio Ambiente (para el periodo comprendido entre 1987-93) y de la Consejería de Medio Ambiente (a partir de 1994). Estos datos aparecen en la Cuadro 15.

Un balance general nos permite comprobar, por un lado, la existencia de un crecimiento de todas las líneas de intervención. Hay que destacar en el periodo 19882003 el incremento registrado por el programa de Conservación y Aprovechamiento de los recursos naturales ${ }^{10}(15,32 \%)$, el programa de Educación Ambiental $(14,46 \%)$ y el programa de Recuperación y Protección de la Calidad Ambiental (13,95\%).

\footnotetext{
${ }^{10}$ Las cifras de 1988 tomadas para analizar el crecimiento recogen las cantidades del programa Conservación y Protección de la Naturaleza y el de Mejora y Aprovechamiento del Patrimonio Natural, ya que a partir de 1999 se unen.
} 
Por otro lado, debemos hacer mención al hecho de que, a medida que pasan los años, algunos programas presentan una estabilidad en cuanto a su existencia y al crecimiento de los recursos asignados: Dirección y Servicios Generales, Recuperación y Protección de la Calidad Ambiental, y Conservación y Aprovechamiento de los recursos naturales (que aglutina a los programas de Mejora y Aprovechamiento del Patrimonio natural, y Conservación y Protección de la Naturaleza). Otros programas se unen a algunos de los citados. También existen programas que aparecen de forma puntual y, o bien desaparecen con posterioridad como tales (por ejemplo, el de Mejora de las estructuras forestales), o bien se convierten en estables a partir de su aparición (por ejemplo, el Programa de Planificación e Información Ambiental).

En la Cuadro 15 encontramos cifras que presentan un crecimiento muy fuerte en determinados años, pero que en realidad se deben a motivos puntuales. Así, en el programa de Dirección y Servicios Generales en 1995 casi se duplica la dotación del año anterior, lo que resulta lógico, dado que son los primeros presupuestos que se diseñan tras la creación de la Consejería de Medio Ambiente en 1994 y ésta debe dotarse de las correspondientes Direcciones y Servicios.

Por otro lado, la cifra del año 1995 del programa de Acciones Integradas para el Ecodesarrollo recoge una partida de 71,52 millones de euros destinados a infraestructuras y equipamientos ambientales, por lo que no se produce un crecimiento tan destacado respecto al año anterior como podría deducirse de la comparación de los datos.

Para concluir el estudio de la distribución por programas, nos referimos a la participación de cada uno de ellos en el total de Gasto Público en medio ambiente (Cuadro 16). Si comparamos los datos de 1988 con los del año 2003, destaca el incremento de los programas de Conservación y Aprovechamiento de los Recursos Naturales (9,96 puntos porcentuales) y de Recuperación y Protección de la Calidad Ambiental $(4,32)$. En el lado opuesto encontramos el programa de Dirección y Servicios Generales, que pasa a representar en 2003 aproximadamente el $50 \%$ de su participación en el total de Gasto Público en medio ambiente en 1988. 

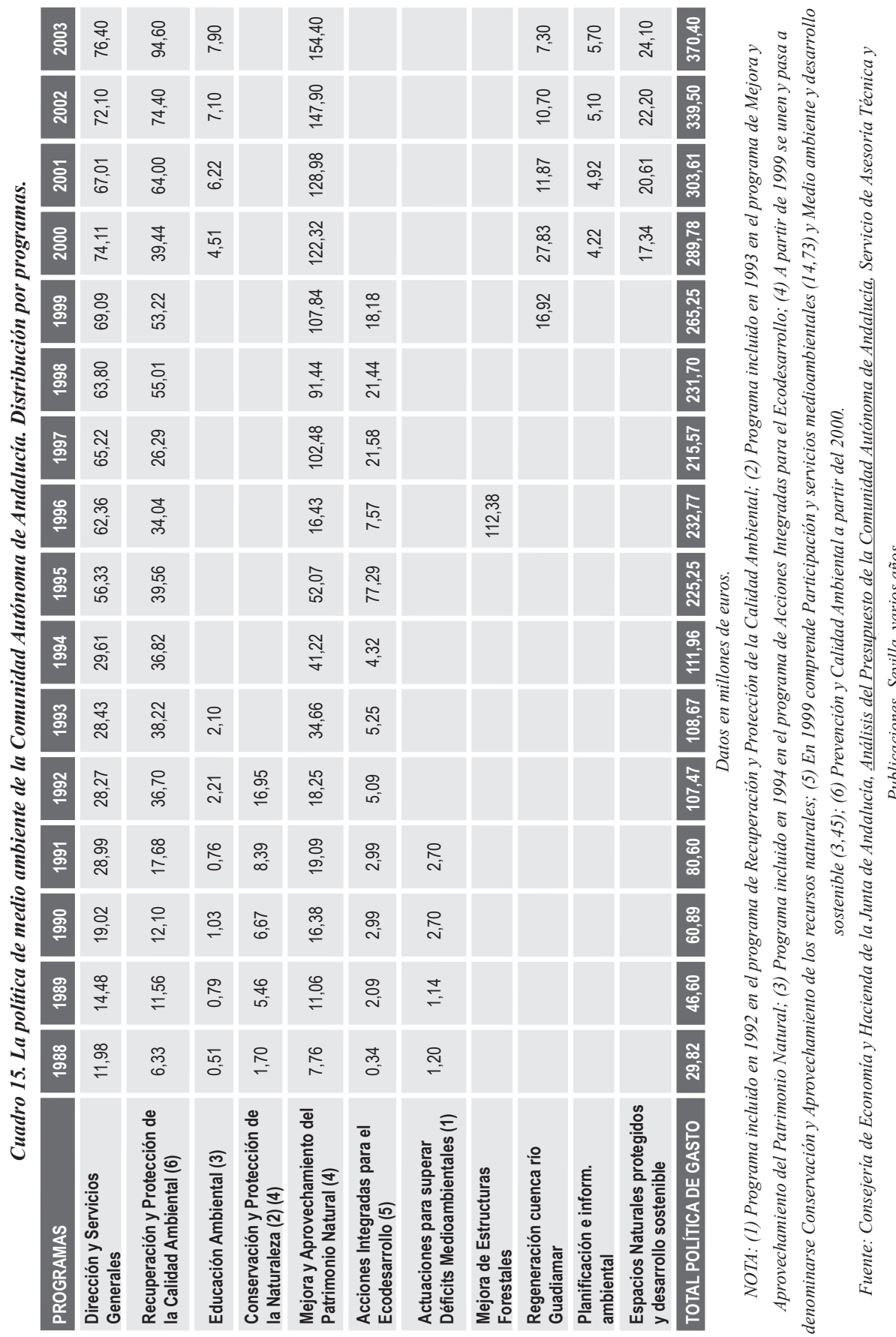


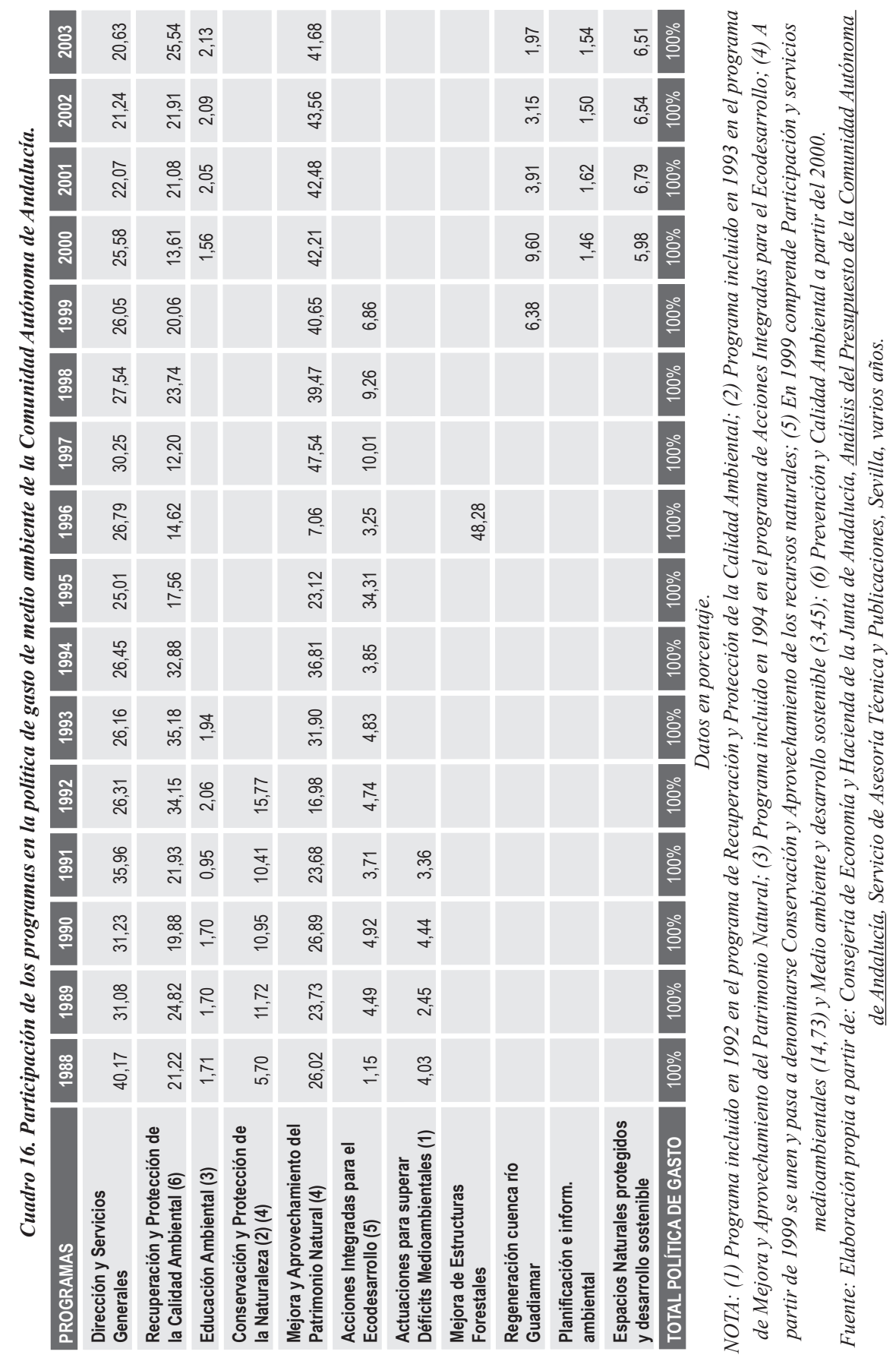


A continuación vamos a estudiar la distribución del Gasto Público en medio ambiente en Andalucía por capítulos presupuestarios. En la Cuadro 17 podemos apreciar cómo desde la década de los noventa se produce una disminución en la partida de gastos corrientes: concretamente si en 1991 se situaban en el 33,25\%, en 2003 representan el 24,30\%, la cifra más baja del periodo. La partida que más ha contribuido a esta reducción ha sido la correspondiente a los Gastos de personal (que baja nueve puntos porcentuales), seguida del capítulo de Gastos de bienes corrientes y servicios (con una bajada superior al 2,1).

Por lo que se refiere a las operaciones de capital, la tendencia en el periodo 1991-2003 es de crecimiento: pasa del 66,75\% a representar el 75,70\% en 2003 . Este crecimiento no se debe a un comportamiento similar entre sus dos componentes: mientras que el capítulo de Inversiones reales pierde protagonismo (al pasar del $64,48 \%$ al $57,5 \%$ ), el capítulo de Transferencias de capital registra un importante crecimiento, ya que se coloca en el $18,2 \%$ partiendo de un $2,27 \%$. No obstante, debemos destacar el elevado volumen de gasto que se destina a las Inversiones reales: en 2003 aproximadamente el $76 \%$ de las operaciones de capital se dirigen a este capítulo. Esto es una prueba del gran esfuerzo que está realizando la Comunidad Autónoma andaluza para superar los déficits que en materia ambiental presenta. 
Cuadro 17. La política de medio ambiente de la Comunidad Autónoma de Andalucía. Distribución por capítulos: estructura porcentual.

\begin{tabular}{|c|c|c|c|c|c|c|c|c|c|c|c|c|c|}
\hline CAPÍTULOS & 1991 & 1992 & 1993 & 1994 & 1995 & 1996 & 1997 & 1998 & 1999 & 2000 & 2001 & 2002 & 2003 \\
\hline I Gastos de Personal & 26,79 & 21,27 & 21,59 & 21,85 & 21,74 & 22,66 & 24,26 & 22,97 & 20,77 & 19 & 19,7 & 18,5 & 17,7 \\
\hline $\begin{array}{l}\text { II Gastos de Bienes } \\
\text { Corrientes y Servicios }\end{array}$ & 6,03 & 5,72 & 4,63 & 5,26 & 3,14 & 4,06 & 4,78 & 4,48 & 3,98 & 3,7 & 3,6 & 4 & 3,9 \\
\hline \multicolumn{14}{|l|}{ III Gastos Financieros } \\
\hline $\begin{array}{l}\text { IV Transferencias } \\
\text { Corrientes }\end{array}$ & 0,43 & 0,32 & 0,3 & 0,28 & 0,09 & 0,09 & 0,11 & 0,19 & 0,16 & 2,2 & 2,4 & 2,5 & 2,7 \\
\hline \multicolumn{14}{|l|}{ V Amortizaciones } \\
\hline $\begin{array}{l}\text { TOTAL OPERACIONES } \\
\text { CORRIENTES }\end{array}$ & 33,25 & 27,31 & 26,53 & 27,39 & 24,97 & 26,8 & 29,14 & 27,64 & 24,91 & 25 & 25,7 & 25 & 24,3 \\
\hline VI Inversiones Reales & 64,48 & 63,64 & 68,96 & 70,91 & 73,66 & 68,97 & 66,59 & 64,84 & 65,36 & 55,4 & 56,7 & 53,9 & 57,5 \\
\hline $\begin{array}{l}\text { VII Transferencias de } \\
\text { Capital }\end{array}$ & 2,27 & 9,05 & 3,71 & 1,41 & 1,2 & 4,22 & 4,27 & 7,51 & 9,73 & 19 & 16,9 & 21,1 & 18,2 \\
\hline $\begin{array}{l}\text { TOTAL OPERACIONES } \\
\text { DE CAPITAL }\end{array}$ & 66,75 & 72,69 & 72,67 & 72,32 & 74,86 & 73,2 & 70,86 & 72,36 & 75,09 & 74,4 & 73,7 & 75 & 75,7 \\
\hline $\begin{array}{l}\text { TOTAL OPERACIONES } \\
\text { NO FINANCIERAS }\end{array}$ & 100 & 100 & 99,19 & 99,7 & 99,83 & 100 & 100 & 100 & 100 & 99,4 & 99,4 & 100 & 100 \\
\hline $\begin{array}{l}\text { TOTAL OPERACIONES } \\
\text { FINANCIERAS }\end{array}$ & 0 & 0 & 0,81 & 0,3 & 0,17 & 0 & 0 & 0 & 0 & 0,6 & 0,6 & 0 & 0 \\
\hline TOTAL POLÍTICA & 100 & 100 & 100 & 100 & 100 & 100 & 100 & 100 & 100 & 100 & 100 & 100 & 100 \\
\hline
\end{tabular}

Fuente: Consejería de Economía y Hacienda de la Junta de Andalucia, Análisis del Presupuesto de la Comunidad Autónoma de Andalucía, Servicio de Asesoría Técnica y Publicaciones, Sevilla, varios años.

\section{ANÁLISIS COMPARATIVO}

En este apartado queremos realizar una comparación entre el Gasto Público en medio ambiente a nivel nacional y el realizado por la Comunidad Autónoma andaluza. Para ello sería interesante comparar la participación del Gasto Público en medio ambiente de Andalucía en el Gasto consolidado en medio ambiente de España, la participación de ambos niveles administrativos respecto al total del Gasto Público respectivo, la distribución de los recursos en capítulos presupuestarios (operaciones corrientes y operaciones de capital) y el reparto entre las diferentes actividades ambientales.

En primer lugar, establecemos la participación del Gasto Público en medio ambiente de la Comunidad Autónoma de Andalucía en el Gasto consolidado en medio ambiente a nivel nacional (Cuadro 18). Se puede observar cómo este indicador también nos muestra un crecimiento continuado en el tiempo (con la excepción de 
1993), por lo que aumenta la participación del Gasto andaluz en medio ambiente respecto al Gasto Público español. Una de las razones la encontramos en el elevado porcentaje de superficie protegida que se encuentra en esta Comunidad Autónoma (el $17 \%$ de la superficie total frente al $7 \%$ en el caso de España), así como en el mayor esfuerzo que debe realizar para superar los déficits medioambientales generados por las distintas actividades productivas.

En la Cuadro 18 a partir de 1996 los datos para España solo reflejan la participación del Gasto Público de la Sección 23 Ministerio de Medio Ambiente en el Gasto Público del Subsector Estado. Si se considerase el gasto ambiental que realizan otras secciones aumentaría esta participación, por lo que esto justifica en parte el descenso registrado a nivel nacional a partir de 1996. Es por ello que para el periodo 1997-2003, como solo disponemos de los datos de Gasto consolidado correspondientes a la Sección 23 Ministerio de Medio Ambiente, debemos interpretar las cifras de participación, no en términos absolutos sino solamente para ver la tendencia que se sigue: desciende la participación del Gasto Público en medio ambiente de Andalucía si lo comparamos con la evolución seguida por la Sección 23.

Cuadro 18. Participación del gasto público en medio ambiente de Andalucía en el gasto consolidado en medio ambiente total (1987-1995) y de la sección 23 Ministerio de Medio Ambiente (1997-2003).

\begin{tabular}{|l|c|c|c|c|c|c|c|c|c|}
\hline & 1987 & 1988 & 1989 & 1990 & 1991 & 1992 & 1993 & 1994 & 1995 \\
\hline ANDALUCÍA & 23,44 & 29,82 & 46,60 & 60,89 & 80,60 & 107,47 & 108,67 & 111,96 & 225,25 \\
\hline ESPAÑA & $1.581,97$ & $1.901,81$ & $2.437,19$ & $2.899,32$ & $3.209,05$ & $3.918,33$ & $4.400,40$ & $3.962,17$ & $4.958,71$ \\
\hline PARTICIPACIÓN & $1,48 \%$ & $1,57 \%$ & $1,91 \%$ & $2,10 \%$ & $2,51 \%$ & $2,74 \%$ & $2,47 \%$ & $\mathbf{2 , 8 3} \%$ & $\mathbf{4 , 5 4 \%}$ \\
\hline
\end{tabular}

\begin{tabular}{|l|c|c|c|c|c|c|c|}
\hline & 1997 & 1998 & 1999 & 2000 & 2001 & 2002 & 2003 \\
\hline ANDALUCÍA & 216 & 231 & 265 & 290 & 304 & 340 & 370 \\
\hline SECCIÓN 23 & $1.657,98$ & $1.825,98$ & $2.065,68$ & $2.236,19$ & $2.494,24$ & $2.678,62$ & $3.001,68$ \\
\hline PARTICIPACIÓN & $13,03 \%$ & $12,65 \%$ & $12,83 \%$ & $12,97 \%$ & $12,19 \%$ & $12,69 \%$ & $12,33 \%$ \\
\hline
\end{tabular}

Datos en millones de euros.

Fuente: Elaboración propia a partir de Agencia de Medio Ambiente (varios años): Informe Medio Ambiente en Andalucía y Subdirección General de Normativa y Cooperación Institucional, Gasto Público en medio ambiente 1995. Análisis comparativo 1987-1995, "Serie estadísticas", Centro de Publicaciones de la Secretaría General Técnica del Ministerio de Medio Ambiente, Madrid, 1988, pág. 29. 
Para conocer cuál ha sido la evolución de la participación del Gasto Público en medio ambiente respecto al Gasto Público total, en la Cuadro 19 aparecen recogidas las participaciones respectivas de Andalucía y España. Aunque aparecen las cifras hasta el año 2003, para el caso de España solo debe tenerse en cuenta el periodo 1987-95, pues a partir de esta fecha los problemas metodológicos ya comentados a nivel nacional distorsionarían el análisis.

De la comparación de la participación del Gasto Público en medio ambiente en Andalucía respecto al Presupuesto de la Junta de Andalucía y la participación del Gasto Público en medio ambiente en España respecto al Gasto Público consolidado total (periodo 1987-95), se deduce que la distancia no se acorta significativamente: tanto en 1987 como en 1994 existe una diferencia de 2,09 puntos porcentuales que pasan a 1,72 en 1995. Esto puede ser debido a que en ese periodo, aunque ambas Administraciones han realizado un esfuerzo, éste ha sido mayor en Andalucía.

Para el periodo 1996-2003, con las salvedades comentadas, vemos que se reducen las distancias debido a la pérdida de participación del Gasto Público en medio ambiente en Andalucía respecto al Presupuesto de la Junta de Andalucía, frente al incremento de la participación del Gasto Público de la Sección 23 en el Gasto Público del Subsector Estado.

Cuadro 19. Participación del gasto público en medio ambiente respecto al gasto público total: el caso de España y Andalucía.

\begin{tabular}{|l|c|c|c|c|c|c|c|c|c|}
\hline PARTICIPACIÓN & 1987 & 1988 & 1989 & 1990 & 1991 & 1992 & 1993 & 1994 & 1995 \\
\hline ANDALUCÍA & $0,58 \%$ & $0,61 \%$ & $0,75 \%$ & $0,82 \%$ & $0,99 \%$ & $1,17 \%$ & $1,10 \%$ & $1,03 \%$ & $2,00 \%$ \\
\hline ESPAÑA & $2,66 \%$ & $2,86 \%$ & $3,19 \%$ & $3,23 \%$ & $3,10 \%$ & $3,53 \%$ & $2,62 \%$ & $3,12 \%$ & $3,72 \%$ \\
\hline
\end{tabular}

\begin{tabular}{|l|c|c|c|c|c|c|c|c|}
\hline PARTICIPACIÓN & 1996 & 1997 & 1998 & 1999 & 2000 & 2001 & 2002 & 2003 \\
\hline ANDALUCÍA & $1,90 \%$ & $1,57 \%$ & $1,56 \%$ & $1,71 \%$ & $1,78 \%$ & $1,77 \%$ & $1,85 \%$ & $1,88 \%$ \\
\hline ESPAÑA & $0,91 \%$ & $1,02 \%$ & $1,15 \%$ & $1,24 \%$ & $1,30 \%$ & $1,24 \%$ & $1,33 \%$ & $1,28 \%$ \\
\hline
\end{tabular}

Nota: A partir de 1996 los datos para España solo reflejan la participación del Gasto Público de la Sección 23 Ministerio de Medio Ambiente en el Gasto Público del Subsector Estado. Si se considerase el gasto ambiental que realizan otras secciones aumentaría esta participación, por lo que esto justifica en parte el descenso registrado a nivel nacional a partir de 1996.

Fuente: Elaboración propia a partir de Agencia de Medio Ambiente (varios años): Informe Medio Ambiente en Andalucía y del Boletín Oficial de la Junta de Andalucía.

Por lo que se refiere a la distribución por capítulos del Gasto Público en medio ambiente (Cuadro 11 para España y 17 para Andalucía), debemos señalar que se produce una reducción de la partida de gastos corrientes tanto en el caso de España 
(de $66,03 \%$ en 1987 a 53,61\% en 1995) como en el de Andalucía (de 33,25\% en 1991 a 24,3\% en 2003), especialmente en los Capítulos de Gastos de personal y de Gastos de bienes corrientes y servicios.

En el caso de los gastos de capital, en España experimentan un incremento, pasando del 33,97\% en 1987 al 46,39\% en 1995. Esto sucede también en Andalucía: las operaciones de capital en el periodo 1991-2003 pasan del 66,75\% a representar el $75,7 \%$, aunque debemos señalar que en este periodo se reduce la participación de las Inversiones reales en el total de Operaciones de capital y se incrementan las Transferencias de capital.

Por último, hay que señalar que no es posible comparar las actividades ambientales a las que se destinan las partidas presupuestarias en España y en Andalucía, ya que no existe una información homogénea.

\section{CONCLUSIONES}

A lo largo de este artículo se ha podido constatar cómo desde la incorporación de España a la Comunidad Europea hemos asistido a un crecimiento prácticamente ininterrumpido en materia de Inversión y Gasto Público en medio ambiente en el Presupuesto de España, a un ritmo superior al registrado por la Inversión real total y el Gasto Público total.

Por capítulos presupuestarios, podemos decir que en el periodo 1987-95 se ha producido una importante reducción de los gastos corrientes (trece puntos porcentuales), aunque las diferentes partidas han sufrido variaciones dispares (disminución de los gastos de personal y los de compra de bienes y servicios, ligero aumento de los gastos financieros y se multiplican por diez las transferencias corrientes). En el periodo 1996-2004, asistimos a un crecimiento de los gastos corrientes, motivado fundamentalmente por el incremento de las transferencias corrientes, ya que los gastos de personal y los gastos de bienes y servicios crecen moderadamente y los gastos financieros se reducen.

En cuanto a los gastos de capital debemos indicar que se han incrementado desde 1987, destacando especialmente la partida destinada a inversiones reales. 
En el caso de la Comunidad Autónoma andaluza, podemos decir que la partida presupuestaria que la Junta de Andalucía dedica a medio ambiente representa un porcentaje pequeño con relación al volumen total del Presupuesto, aunque en los últimos años ha experimentado un importante crecimiento. Esto se traduce en un aumento de la participación del Gasto Público en medio ambiente en el Gasto Público de la Junta de Andalucía. Por otro lado, también es cierto que el Gasto Público en medio ambiente en Andalucía crece con relación al Gasto consolidado en medio ambiente en España.

En cuanto a la distribución del Gasto en medio ambiente por capítulos presupuestarios, en Andalucía asistimos a lo largo del periodo 1991-2003 a una reducción de los gastos corrientes y a un crecimiento de las operaciones de capital. Debemos destacar el elevado volumen de gasto que se destina a las inversiones reales: aproximadamente el $76 \%$ de las operaciones de capital se dirigen a este capítulo. Este porcentaje es similar al que encontramos a nivel nacional (82\%) y es una prueba del gran esfuerzo que se está realizando con el objetivo de superar los déficits que en materia ambiental presenta tanto la Comunidad Autónoma andaluza como España.

\section{BIBLIOGRAFÍA}

Consejería de Economía y Hacienda de la Junta de Andalucía, Análisis del Presupuesto de la Comunidad Autónoma de Andalucía para 1991, Servicio de Estudios y Publicaciones de la Consejería de Economía y Hacienda, Sevilla, 1991.

Consejería de Economía y Hacienda de la Junta de Andalucía, Análisis del Presupuesto de la Comunidad Autónoma de Andalucía para 1992, Servicio de Asesoría Técnica y Publicaciones, Sevilla, 1992.

Consejería de Economía y Hacienda de la Junta de Andalucía, Análisis del Presupuesto de la Comunidad Autónoma de Andalucía para 1993, Servicio de Asesoría Técnica y Publicaciones, Sevilla, 1993.

Consejería de Economía y Hacienda de la Junta de Andalucía, Análisis del Presupuesto de la Comunidad Autónoma de Andalucía para 1994, Servicio de Asesoría Técnica y Publicaciones, Sevilla, 1994. 
Consejería de Economía y Hacienda de la Junta de Andalucía, Presentación Proyecto del Presupuesto de la Comunidad Autónoma de Andalucía para 1995, Servicio de Asesoría Técnica y Publicaciones, Sevilla, 1994.

Consejería de Economía y Hacienda de la Junta de Andalucía, Presentación Proyecto del Presupuesto de la Comunidad Autónoma de Andalucía para 1996, Servicio de Asesoría Técnica y Publicaciones, Sevilla, 1995.

Consejería de Economía y Hacienda de la Junta de Andalucía, Análisis del Presupuesto de la Comunidad Autónoma de Andalucía para 1997, Servicio de Asesoría Técnica y Publicaciones, Sevilla, 1996.

Consejería de Economía y Hacienda de la Junta de Andalucía, Análisis del Presupuesto de la Comunidad Autónoma de Andalucía para 1998, Servicio de Asesoría Técnica y Publicaciones, Sevilla, 1997.

Consejería de Economía y Hacienda de la Junta de Andalucía, Análisis del Presupuesto de la Comunidad Autónoma de Andalucía para 1999, Servicio de Asesoría Técnica y Publicaciones, Sevilla, 1998.

Consejería de Economía y Hacienda de la Junta de Andalucía, Análisis del Presupuesto de la Comunidad Autónoma de Andalucía para 2000, Servicio de Asesoría Técnica y Publicaciones, Sevilla, 1999.

Consejería de Economía y Hacienda de la Junta de Andalucia, Presentación Proyecto de Ley del Presupuesto de la Comunidad Autónoma de Andalucía 2001, Servicio de Asesoría Técnica y Publicaciones, Sevilla, 2000.

Consejería de Economía y Hacienda de la Junta de Andalucía, Análisis del Presupuesto de la Comunidad Autónoma de Andalucía para 2002, Servicio de Asesoría Técnica y Publicaciones, Sevilla, 2002.

Consejería de Economía y Hacienda de la Junta de Andalucía, Análisis del Presupuesto de la Comunidad Autónoma de Andalucía para 2003, Servicio de Asesoría Técnica y Publicaciones, Sevilla, 2003.

Dirección General de Calidad y Evaluación Ambiental, Medio Ambiente en España 1996, "Serie memorias", Centro de Publicaciones de la Secretaría General Técnica del Ministerio de Medio Ambiente, Madrid, 1997. 
Dirección General de Planificación y Participación, Informe Medio Ambiente en Andalucía, Junta de Andalucía, Sevilla, varios años.

Lozano Oyola, M. y Romero Landa, L.B., Sector Público y Medio Ambiente (Anexo, págs. 761-770), del libro Economía Andaluza, Algaida, Sevilla, 1997.

Ministerio de Obras Públicas, Transportes y Medio Ambiente, Gasto Público en Medio Ambiente en 1991 y datos comparativos 1987-1991, Centro de Publicaciones del MOPTMA, Madrid, 1994.

Subdirección General de Normativa y Cooperación Institucional, Gasto Público en medio ambiente 1995. Análisis comparativo 1987-1995, "Serie Estadísticas", Centro de Publicaciones de la Secretaría General Técnica del Ministerio de Medio Ambiente, Madrid, 1998. 